\title{
Response to Letter to the Editor
}

Dr. Rafael's independent thinking should be applauded, both for his dissenting view of the etiology of $\mathrm{AD}$ and for his pioneering work with omental transplantation for the treatment of neurological conditions. It is questionable, however, if our case of cryptococcal meningitis (CM) indeed supports his hypothesis that $\mathrm{AD}$ has a microvascular origin. Whereas CM may compromise the intracranial vessels, there was no evidence in our case other than cognitive changes that there was vascular involvement. Angiography or cerebral blood flow studies were not done, and there are no biopsy or autopsy results (the patient is alive and well in Wisconsin). Although his MRI scan did show microvascular ischemic changes, they were judged at the time to be nonspecific and age-related, and no comments were made regarding an atypical distribution. Unfortu- nately, the contrast-enhanced MRI study was of limited quality, and all of the films were recycled years ago, making further review impossible. It is therefore very difficult to say if our case strengthens Dr. Rafael's hypothesis. If nothing else, it certainly fosters continued discussion.

Tom Ala, M.D.

Center for Alzheimer Disease and Related Disorders Southern Illinois University School of Medicine

P.O. Box 19643

Springfield, Illinois 62794-9643

E-mail: tala@siumed.edu 